(C) [2009] IEEE. Reprinted, with permission, from [Quang Vinh Nguyen, Mao Lin Huang, Large Scale Network Analysis with Interactive Visualisation, 2009 Sixth International Conference on Computer Graphics, Imaging and Visualization, 2009]. This material is posted here with permission of the IEEE. Such permission of the IEEE does not in any way imply IEEE endorsement of any of the University of Technology, Sydney's products or services. Internal or personal use of this material is permitted. However, permission to reprint/republish this material for advertising or promotional purposes or for creating new collective works for resale or redistribution must be obtained from the IEEE by writing to pubs-permissions@ieee.org. By choosing to view this document, you agree to all provisions of the copyright laws protecting it 


\title{
Large Scale Network Analysis with Interactive Visualisation
}

\author{
Quang Vinh Nguyen ${ }^{1}$, Mao Lin Huang ${ }^{2}$ \\ ${ }^{1}$ School of Computing \& Mathematics, University of Western Sydney \\ ${ }^{2}$ Faculty of Engineering \& Information Technology, University of Technology, Sydney \\ \{vinh@scm.uws.edu.au,maolin@it.uts.edu.au\}
}

\begin{abstract}
This paper proposes a new interactive visualisation for analysing large hierarchical structures and networks. The technique combines of different graph layout methods with a layout refinement process, an interactive navigation mechanism and clustering algorithms. The integration of these components makes it flexible in dealing with a variety of graph and hierarchical structures. Interactive exploration is enabled with chaincontext view. We aim to provide user with an effective mechanism for understanding of the nature of various networks. This could lead to the discovering and revealing of the hidden structures and relationships among elements as well as relationships associated with the elements.
\end{abstract}

Keywords--- Networks, Information Visualisation, Interaction, Visual Analytics.

\section{Introduction}

Our knowledge-driven technology-mediated environments generate more than 5 exabytes $\left(5 \times 10^{18}\right.$ bytes) of new information each year [1]. Much of this information reflects the complex interconnected processes and systems that operate in these environments that span across ecological micro- and macro-systems to interconnected financial markets. A network, such as a social network, can be defined as the information associated with and the relationships between actors which are elements in the network, such as people, groups, corporate organisations, business partners, and others. Considering such systems in isolation is not longer feasible. For the last decade, there has been a rapidly increasing interest in modelling and understanding such systems and processes through network models [2]. Real world networks are often very large with thousands or even millions of elements and relationships. Examples of such networks range from biological and ecological networks through to sociotechnological networks, such as telephone call graphs and the underlying interaction graphs on the Internet [3].
These heterogeneous networks present big challenges on how to discover reliable and significant patterns and irregularities within them? How do we holistically make sense of these discoveries and their network models? How do we harness these data at different levels of granularity in collective decision making processes? The challenge - and the fundamental focus of this project resides in creating a fast and effective platform in which analysts quickly analyse huge amount of data as well as share their insights and knowledge.

Network analysis brings a lot of benefits in a wide range of application domains, such as economics/finance analysis [4], homeland defence [5], and knowledge management, organisation development, social science, health informatics (see [6]). For example, analysing a terrorism network can help to understand the roles and relational structures among terrorists, their organisations, the associated events and other information that could reveal the potential vulnerabilities and identify any potential attack before it occurs. Another example is that the social network analysis for large cooperate organisations could identify the unofficial organisational roles, such as central connector, boundary spanner, information broker and peripheral specialists. The analysis of social network in organisations can provide business benefits including 1) identify and retention with vital knowledge and connections, 2) improvement of innovation, productivity and responsiveness, 3) lead to better decisions about organisational changes and establishment of key knowledge roles, and 4) insight into challenges of knowledge transfer and integration following restructuring, merges or acquisitions.

As a result, large networks visualization has recently received a lot of attention of researchers in supporting the visual analytics. There are a number of good techniques that are quite capable of visualizing large graphs of thousands to hundred thousands of nodes and edges, named a few [8-11]. Research in how to effectively integrating interactive visualisations with sense-making sequence (see Figure 1) is still one of the open topics in visual analytics.

The interactive visualisation helps the analyst to gain knowledge of the data through our powerful human visual perception, ideally driving the system toward 
more focused and more adequate analytical techniques. Visualisation should provide not only the simplified abstract view of the entire network, but it also display in detail information at a particular focus point. Dually, the visualization should also allow analysts to interact and explore to make further discovery of and insight into the information; from which they can obtain a better understanding of the data and structures. From the new knowledge and discovery, the analysts can evaluate and refine automated analysis as well as interactive visualisation processes (see Figure 1).

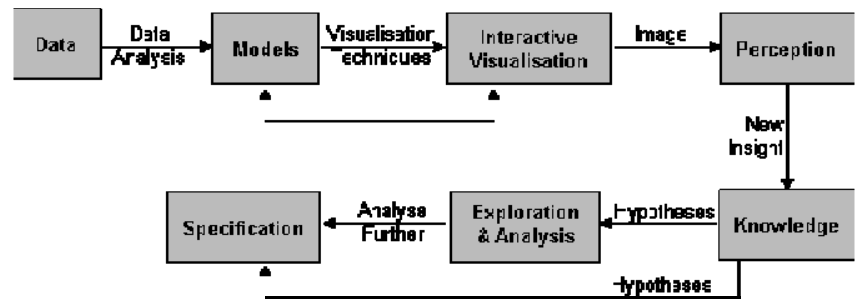

Figure 1. The process sequence of Visual Analytics based on sense-making loop (Adapted from [12])

This paper presents an ongoing research work for interactively visualising various large graphs and networks. The visualisation is integrated at the sensemaking loop to enable the visual analytics of large data sets.

\section{The Architecture of Visualisation}

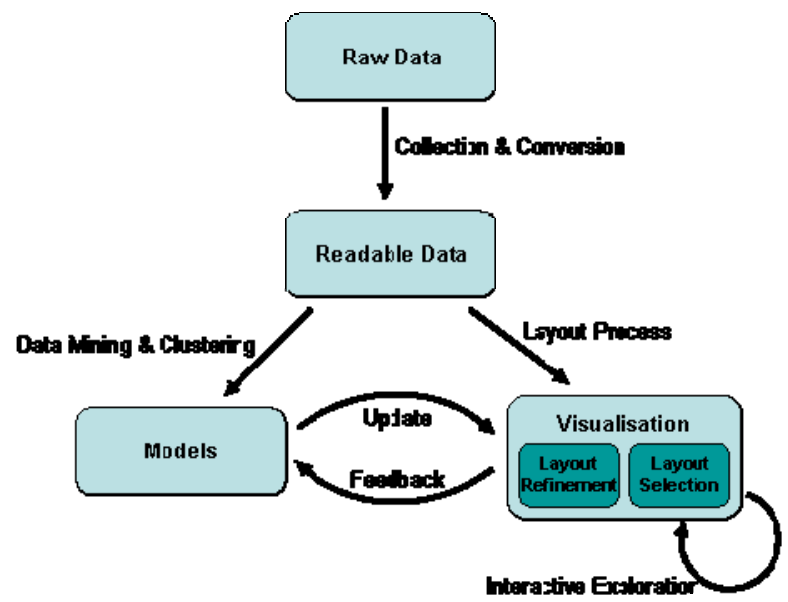

\section{Figure 2. The architecture of Visualisation}

The visualisation system includes three main components including data conversion, data clustering and data mining, and interactive visualization (see Figure 2). The three components are briefly described as below.

Collection and conversion - We collect data from a variety of sources, such as email networks, protein networks, citation networks, and others. This process is responsible for converting data from different format into uniform XML format for further analysis and visualisation.
Data Mining and Clustering - This process analyses structures of networks and finds the clusters of similarity and connection based on algorithms. At the current progress of this project, we only use clustering to discover similar structures in the networks. We use a graph clustering method which can quickly discover the community structures embedded in large graphs and divide the graph into densely connected sub-graphs. The graph clustering algorithm divides the graphs into smaller and smaller sub-graphs based on the density of connection within and between subgroups. Although this process does not require a very fast algorithm, it should be able to run on a workstation within a reasonable time The computational cost of the clustering algorithm should be controlled with the worst-case running time $\mathrm{O}\left(\mathrm{n}^{2}\right)$ or faster on a sparse graph to ensure its capability of handling hundreds thousands of items within a few hours by using a ordinary personal computer.

This project used both fast graph clustering methods $[13,14]$ which can quickly discover the community structure embedded in large graphs and divide the graph into densely connected sub-graphs. Algorithm in [13] can also achieve a consistent partitioning result in which a graph is divided into a set of clusters of the similar size while algorithm in [14] can be used to discover communities with various size. The clustering process works independently with the visualisation so that the finding can be updated as well as receiving feedback from visualisation.

Visualisation - the visualization interface allows users to view, interact and choose different layout algorithms. A combination of a number of layout algorithms is employed to draw different clusters which aims to optimize the geometrical space and so that a large network with multiple clusters can be drawn at a normal screen size. This interactive interface is able to not only show the abstract view of the entire network with its clustering property and relationships but also display the detail view of any particular group, item and their associate connections and properties.

During the navigation, we allow users to interactively adjust views, and layouts of the network to reach an optimized representation of the graph; from which users can obtain the best understanding of the data and structures they are currently viewing. The visualization involves the real time human-computer interaction, fast graph layout and navigation algorithms for visual processing of hundred thousands of items within minutes or seconds using a personal computer with limited display space and computational power.

The final display is created through the view navigation and graphical attributing. We use rich graphic attributes to assist viewers to quickly identify the domain specific properties associated with data items.

\section{Graph Visualisation}

We have utilised a number of layout algorithms to produce the effective visualisation of large graphs with 
several thousands of nodes and edges. At the current stage, two layout algorithms are applied in our implementation including an adapted algorithm described in [15] and a circular drawing.

\subsection{Layout algorithms}

We apply an enclosure partitioning algorithm, which is similar to the algorithm described in [15], to the drawing of those non-leaf sub-graphs, while a modified Spring Embedder algorithm [16] is applied to the drawing of leaf sub-graphs. This is because the space utilisation and the computational cost issues are crucial when displaying large email networks with thousands or even hundred thousands of nodes and edges in conjunction with the limitation of screen pixels. On the other hand, those leaf sub-structures contain only a small number of nodes so that the space utilization issue becomes less important. Therefore, the aesthetic niceness and flexibility issues need to be more considered. The visualization also displays a high-level node-link diagram to present the overall clustering structure explicitly.

A view refinement process is then applied onto the display to enhance the quality of views, such as the aesthetical niceness and the clearity of cluster properties. The first step is to use a modified Spring Embedder algorithm to refine the layout. This enhances the distribution of nodes based on their local layout and inter-connection with other nodes from other clusters. The second step is to reduce the overlapping among nodes. This works by moving overlapped nodes slightly in the vertical or horizontal directions.

Figure 3 shows an example of the visualization of the entire Enron e-mail network which is the dataset collected from the University of Southern California (including 252,759 messages, 151 employees, 2,064,442 recipients with $500 \mathrm{MB}$ of text). Filtering technique has been applied to simplify the overcrowded display. Figure 4 is an example of a substructure of a protein network where (a) shows the original layout and (b) shows the layout after the view refinement process. This figure indicates that nodes are better distributed in (b) in terms of their connectivity compared to (a). Figure 5 presents the display of a network associated with a selected item whose layout has been refined to reduce node overlapping.

During the exploration, user can switch between different layouts and vice versa. At this stage, we are currently providing two layouts in which user can switch between a typical layout and circular layout. Figure 6 shows the display of the same structure as shown in Figure 4. This is an initial step for a further enhancement. The future implementation aims to provide users with an intelligent visualization that can produce the most suitable displays by interactively providing corresponding layouts and interactions based on the nature of applications, data sets, analyst preference and underline sense-making rules. The visualisation can be adjusted via analyst's feedbacks during the interaction.

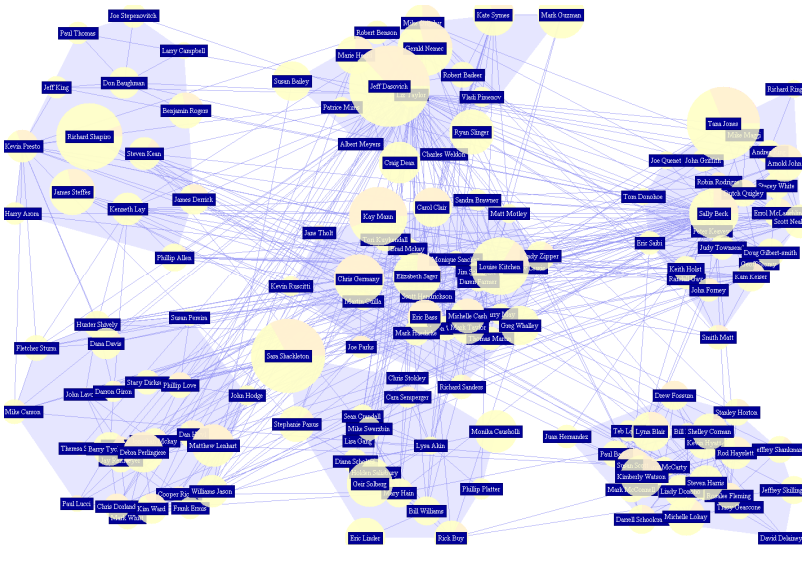

Figure 3. An example of an entire email network visualization of the company: Enron Copus.

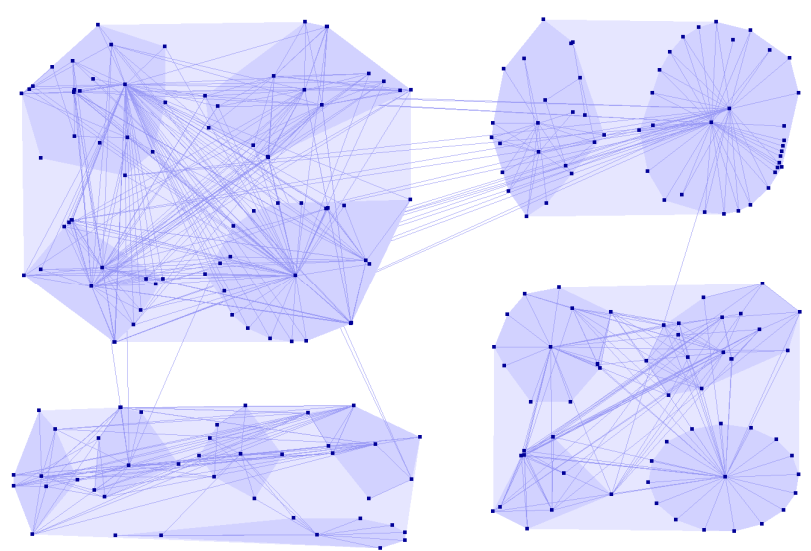

(a)

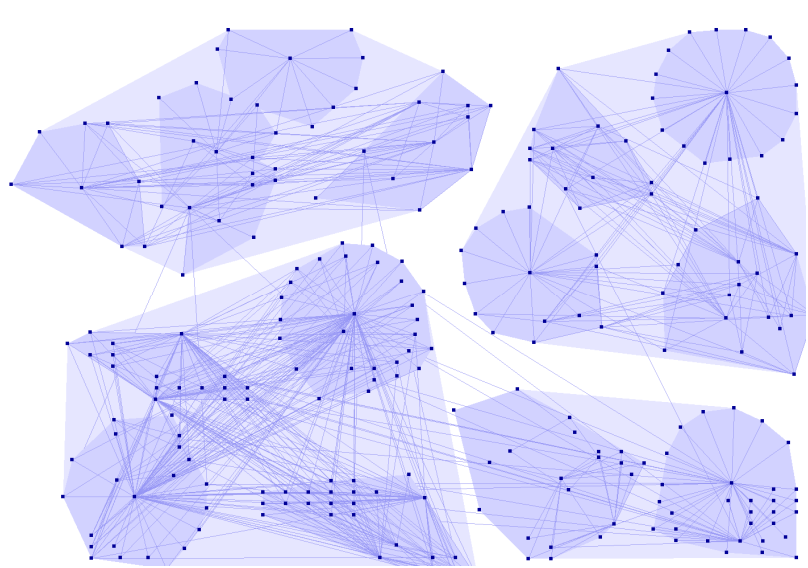

(b)

Figure 4. An example of visualizing a substructure of the protein network, where a) the layout before the view refinement and $b$ ) the layout after the view refinement

\section{Interactive Exploration}

There is no pure visualization technique that could assist data retrieval without providing users with an associate navigation mechanism in graphic user interface design. 
In our visualisation, during the navigation user can

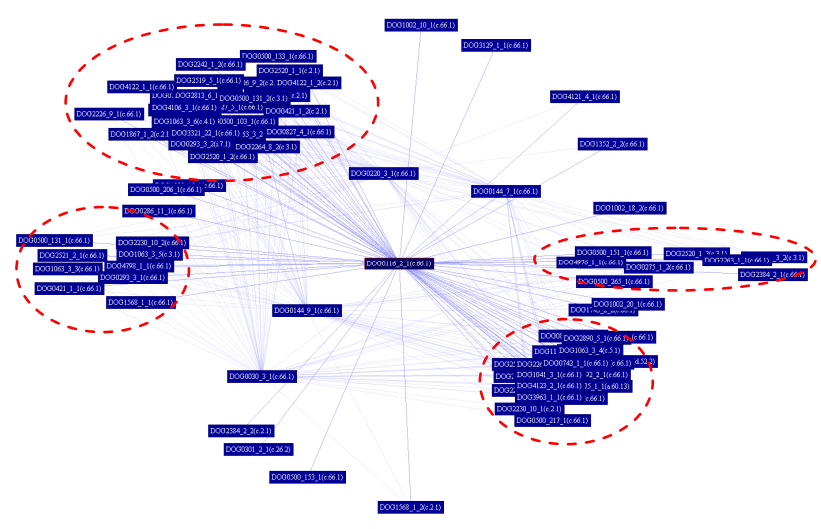

Figure 5. The visualization of a network with reduced node overlaps

representation of the graph; from which user can obtain the best understanding of the data and its relational structures he/she are currently interesting in.

We use an efficient interaction method called chaincontext view [18] for the navigation of large structures. Instead of displaying a single or small number of context views as in traditional approaches, we provide user with the display of a progressive sequence of context views. This approach aims to maintain all contextual information during the navigation. The display of rich context information produced in the exploration path could greatly increase the accuracy of user decisions and reduce the unsuccessful trips and unnecessary views during visual exploration of large hierarchies. This also allows user to trace each step of his/her interaction and makes it easy to jump or return to any level of the hierarchy that he/she has already visited. The latest development is that we produced a fisheye interaction technique, as an alternative approach, for users to browse through the chain-context view. The user is now enabled to enlarge the size of any context view in the chain dynamically to see the detail. Fish-eye is applied to allow a quick browse and enlarge to show the detail of any focus views at any context level. A focus section is defined at a current mouse pointer in which the position and size of all context views in the chain are then adjusted accordingly.

Figure 7 shows an example of the visualisation at a particular stage of the exploration. The left-panel displays the sequence of all context views in which the current focus view is high-lighted for further analysis or return back to that point. The right-panel displays the focus sub-network associated with a person named "Jeff Dasovich".

\section{Attributed Visualisation}

Rich graphical attributes are also employed to assist viewers to quickly identify the domain specific

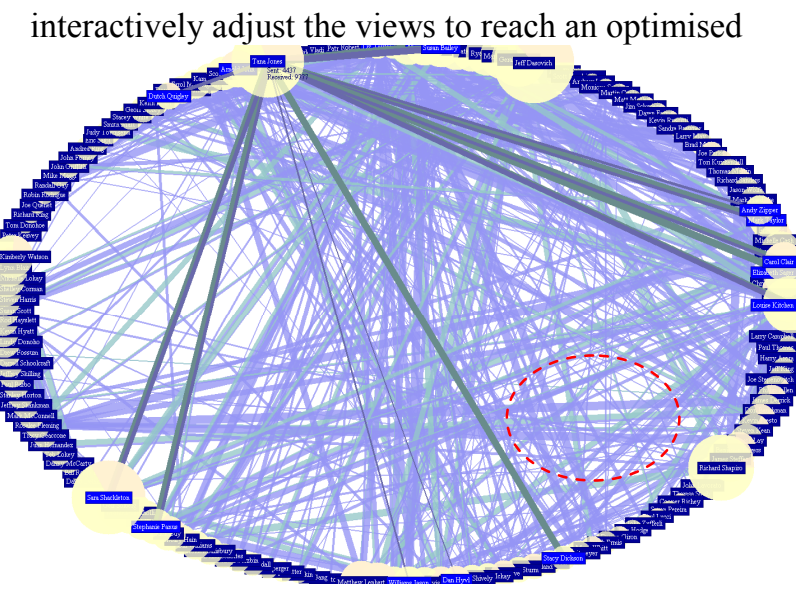

Figure 6. A circular display of the entire email
network collected from Enron Copus (same as
Figure 3 ).

properties of data, social relationships and the cluster structure.

First, background colours were used to assist viewers to quickly identify the hierarchical structure of the clusters. In our prototype, the local regions of nodes at different levels are painted with a same colour but at different brightness (see Figures 3 and 4). This drawing property aims to provide a pleased view while retaining the clarity between sub-clusters.

Node and edge attributes are used to illustrate the property of the elements and their connections. For example, figure 7 shows a network associated with the employee "Jeff Dasovich". The edge thickness represents the proportional strength of sending and receiving emails between employees. The edges representing "sendemails" are painted with violet colour while the edges representing "receive-emails" are painted with darkgreen colour. Arrows can be used to indicate the direction of the connection when required.

\section{Conclusion and Future Work}

We have presented an ongoing technique for the interactive visualization of large networks. Our technique is quite capable of not only displaying the whole network but also allowing users to navigate for further analysis of the sub-networks associated with particular elements from the network. The visual analysis would improve the user understanding of networked data. We believe that this technique will be a promising tool for visual network analysis.

Next, we will improve our visualization system by fully integrating the data mining algorithms into the visualization so that the automated analysis can interact with the visualization and vice versa. An intelligent visualizations is a crucial for future development because it can produce the most suitable display by interactively providing corresponding layouts and interactions based on the nature of applications, data sets, analyst preference and underline sense-making rules. The 
visualisation can also be adjusted via analyst's feedbacks

during the interaction.

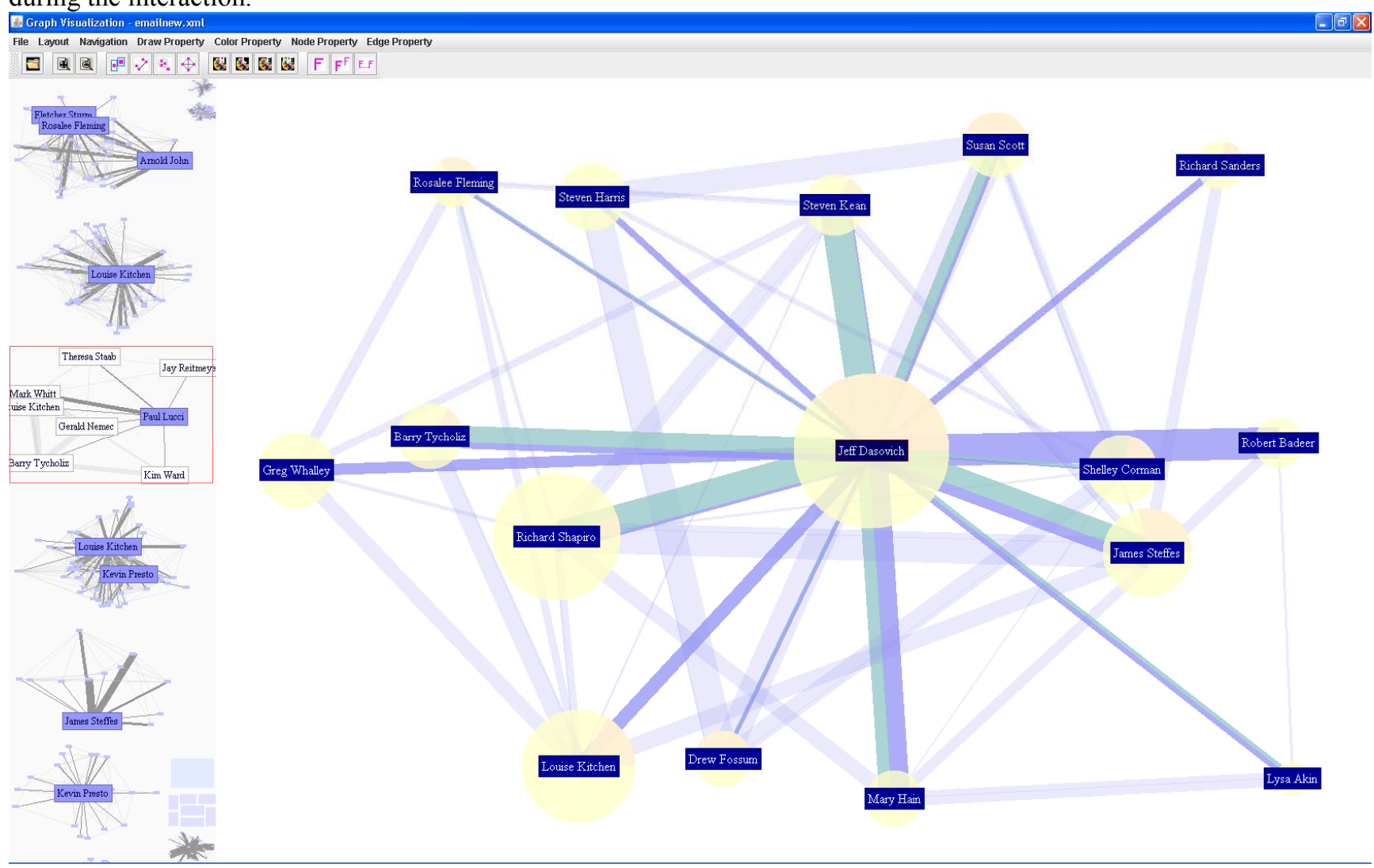

Figure 7. An example of the display at a particular stage during the visual exploration, and we can see that the context-Chain is displayed

\section{References}

[1] Lyman P, Varian HR, "How Much Information?" University of California at Berkeley. http://www2.sims.berkeley.edu/research/projects/howmuch-info-2003/. (Accessed 20 Dec 2008)

[2] Newman, M. Watts, D., Barabási A.-L. The Structure and Dynamics of Networks, Princeton University Press, 2006.

[3] Almaas, E. and Barabási A.-L., "The Architecture of Complexity: From the WWW to Cellular Metabolism", In Proc. Dynamics of Complex Interconnected Systems: Networks and Bioprocesses. NATO Sciences Series, 2006.

[4] Malhotra, Y., "Enabling Knowledge Exchanges for EBusiness Communities", Information Strategy: The Executive's Journal, 2005, pp. 26-31.

[5] Ressler, S., "Social Network Analysis as an Approach to Combat Terrorism: Past, Present, and Future Research", Homeland Security Affairs, 2(2), 2006.

[6] Patton, S., "Social Network Analysis Helps Maximize Collective Smarts", http://www.cio.com/article/print/6956., 2005 (Accessed 20 Dec 2008).

[7] Shen, Z., Ma, K.L. and Eliassi-Rad, T., "Visual Analysis of Large Heterogeneous Social Networks by Semantic and Structural Abstraction", IEEE Transactions on Visualization and Computer Graphics, 12(6), 2006, pp. 1427-1439.
[8] Auber D., "Tulip: a Huge Graph Visualization Framework, Graph Drawing Software", Mathematics and Visualization, 2003, pp. 105-126.

[9] Harel D, Koren Y., "Graph Drawing by HighDimensional Embedding", Journal of Graph Algorithms and Applications, 2004, 8(2): 195-214.

[10] Walshaw C., "A Multilevel Algorithm for ForceDirected Graph Drawing", Mathematics Research Report 00/IM/60, University of Greenwich, 2000.

[11] Abello J, van Ham F, Krishnan N., "ASK-GraphView: a Large Scale Graph Visualization System", IEEE Transactions on Visualization and Computer Graphics 2006, 12(5).

[12] Keim D. et al, "Visual Analytics: Definition, Process, and Challenges Information Visualization”. LNCS 4950, pp. 154-175, 2008.

[13] Huang M.L. and Nguyen Q.V., "A Fast Algorithm for Balanced Graph Clustering", in Proc. $11^{\text {th }}$ Int'l. Conf. on Information Visualisation (IV'07), IEEE, 2007, 46-52.

[14] Newman M.E.J., Girvan M., "Finding and Evaluating Community Structure in Networks", Physical Review E 2004 69:026113.

[15] Huang, M. L. and Nguyen, Q. V. "Large Graph Visualization by Hierarchical Clustering", Journal of Software, 19(8), 2008, pp. 1933-1946.

[16] Eades, P., "A heuristic for graph drawing", Congressus Numerantium, Vol 42, pp. 149-160, 1984. 
[17] Heer, J. "Exploring Enron: Visual Data Mining of E-mail”, http://jheer.org/enron/ (accessed 20 Dec 2008).

[18] Huang, M. L., Nguyen, Q. V., "Space-Filling Interaction with Chain-Context View", IEEE Symposium on Information Visualization 2008 (InfoVis 2008) - Poster Section, IEEE, Columbus, Ohio, October 2008. 